\title{
An Enhanced Group Mobility Protocol for 6LoWPAN-Based Wireless Body Area Networks
}

\author{
Yuh-Shyan Chen \\ Department of CSIE \\ National Taipei University \\ Taipei 237, Taiwan, R. O. C. \\ Email: yschen@mail.ntpu.edu.tw
}

\author{
Chih-Shun Hsu \\ Department of Info. Management \\ Shih Hsin University \\ Taipei 116, Taiwan, R. O. C. \\ Email: cshsu@cc.shu.edu.tw
}

\author{
Hau-Kai Lee \\ Institute of Comm. Eng. \\ National Taipei University \\ Taipei 237, Taiwan, R. O. C. \\ Email: socoolchildren@gmail.com
}

\begin{abstract}
The IPv6 over Low power Wireless Personal Area Network (6LoWPAN) has attracted lots of attention recently because it can be used for the communications of Internet of things (IoT). In this paper, the concept of group-based network roaming in Proxy Mobile IPv6 (PMIPv6) domain is considered in the 6LoWPAN-based wireless body area networks. PMIPv6 is a standard to manage the network-based mobility in allIP wireless network. However, it does not perform well in group-based body area networks. To further reduce the handoff delay and signaling cost, an enhanced group mobility scheme is proposed in this paper to reduce the number of control messages, including Router Solicitation (RS) and Router Advertisement (RA) messages as opposed to the group-based PMIPv6 protocol. Simulation results illustrate that the proposed handoff scheme can reduce the handoff delay and signaling cost. The packet loss ratio and the overhead can also be reduced.
\end{abstract}

Index Terms-Body wireless area networks; Proxy MIPv6; handoff delay; signaling cost; 6LoWPAN.

\section{INTRODUCTION}

Various wireless sensor nodes can be attached to the human body or clothes and hence can form a wireless network named as the Wireless Body Area Networks (WBAN) [1]. These tiny sensors are used to measure particular parameters of the human body, such as the body temperature, blood glucose, pulse rate and heart-beat. These sensing values can be gathered and transmitted to the monitoring server for healthcare applications or surveillance systems.

IPv6 is an effective solution because of the large address spaces and better auto-configuration mechanisms. Since Lowpower Wireless Personal Area Networks (LoWPANs) can support the communications of the Internet of Things (IOTs), it has attracted lots of attention recently. The Internet Engineering Task Force (IETF) has set up a working group for IPv6 over Low power Wireless Personal Area Network (6LoWPAN) [2], which is carried out over IEEE 802.15.4 interfaces. Since the maximum packet size of IEEE 802.15 .4 is 127 bytes, the sensors are unable to hold the complete IPv6 address. The limitation of the packet size is to maintain the low power consumption of sensors, which are powered by batteries only. As a result, 6LoWPAN adds an adaption layer to implement the seamless connection of MAC and network layer. Taking the characteristics of 6LoWPAN into account, the host-based mobility approach is unsuitable to be applied in IP based
Wireless Sensors Networks (IP-WSN) since there are huge amount of tunneling, especially in the case of the WBAN mobility scenario. All of the sensors should have a mobility stack, such as Mobile IPv6, FMIPv6 [3], HMIPv6 [4]. However, the sensors should actively participate in mobility-related signaling that the above protocols are not suitable for them. The network-based mobility protocol would be more suitable for this situation. Proxy Mobile IPv6 (PMIPv6) [5] currently is being standardized by the working group of IETFs Networkbased Localized Mobility Management protocol (NetLMM). PMIPv6 can be considered as the most suitable manner to manage the mobility of the 6LoWPAN-based WBAN.

The sensors' signaling the mobility related message to the agent themselves is a heavy burden. Hence, reducing the signaling cost becomes an important issue because most of the sensors are powered by battery only. Using network-mobility scheme for mobile sensors is a proper solution because it can reduce signaling cost. When the sensors change the point of attachment, there is a delay time before obtaining the IP configuration. Therefore, how to decrease the times of exchanging the control messages in case that a number of sensors attach on one MAG is also an important issue.

To solve the above problem, a new format of control messages for carrying many other identifiers in one message is proposed in this paper so as to reduce the numbers of the control messages and shorten the handoff delay. An enhanced group-based handoff scheme, which adopts the new formatted control messages, is proposed. The proposed handoff scheme contains three phases, namely the registration, up-link handoff, and down-link handoff phases. Simulation results have shown that the proposed protocol can reduce the signaling cost and handoff latency for mobile sensors and it can also decrease the overhead of the Mobile Access Gateway (MAG) in PMIPv6.

The rest of this paper is organized as follows: Section II describes the related works and motivation. In section III, the system model and basic idea are described. Section IV describes the proposed group-based protocol in 6LoWPANbased WBAN. Section V presents the simulation results. Section VI concludes this paper. 


\section{RELATED WORKS}

In this section, related works are described first followed by the motivation of this paper.

\section{A. Related Works}

Istepanian et al. [6] indicates that 6LoWPAN is quite suitable for WBSN since the sensors are based on IEEE 802.15.4 standard which is low power and low data rate. The mobility of 6LoWPAN has been an important solution in wireless communication particularly in WBSN. Most of the existing mobility protocols [7] [3] [4] for IPv6 are not suitable for 6LoWPAN because they are the tunnel-based mobility protocols, which indicates that the mobile sensor nodes need to send lots of control message in order to ensure the continuity of communications. PMIPv6 [5] network-based mobility protocol is a solution to handle the mobility management of the body sensors. The network side performs the mobilityrelated signaling of the sensors and there is no need to perform Duplicate Address Detection (DAD) of the IP address.

J. H. Kim et al. [8] proposed a 6LoWPAN mobility scheme based on Network Mobility (NEMO) [9], which uses a mobile router to support the handoff and modify the dispatch of 6LoWPAN, but the loading of the mobile router is heavy. Since the issue of maintaining the sessions meanwhile reducing the handoff delay and signaling cost of group-based mobility in 6LoWPAN-based WSBN is important, Li et al. [10] provided a scheme for PMIPv6 environment. This scheme considered the case of many correlated sensor nodes moving together and taking handoffs at the same time. The Localized Mobility Anchors (LMA) calculates the SNR value of each sensors and classify the sensors with similar value into groups. This protocol can reduce the handoff signaling cost by sending the PBA (Proxy Binding Ack) message per group. PBU (Proxy Binding Update) and deregistration PBU can decrease the handoff delay by simplifying the procedure. However, the control message of router solicitation (RS) and router advertisement (RA) can not be reduced in case that body sensors move in the PMIPv6 domain. Besides, this scheme is not suitable for the 6LoWPAN-based WSBN.

\section{B. Motivation}

In WBANs, the sensors always move together and take handoff at the same time. For example, a patient may walk around in the hospital for inspections or surgeries. Hence, how to achieve the seamless handoff scheme with less delay time is an important issue. The existing group-based protocol [10] relies on the first newly attaching node to carry the rest of nodes' binding information to reach the goal of reducing the signaling cost and handoff delay. However, the sensors equipped on the human body always attach to the newly access link at the same time. Hence, it is better to use one control message (RS and RA) to carry the whole body sensors information for reducing the signaling cost. Grouping the body sensors to enhance handoff procedure is also a feasible solution. To achieve the goals of reducing the delay time and signaling cost during the handoff procedure, the enhanced

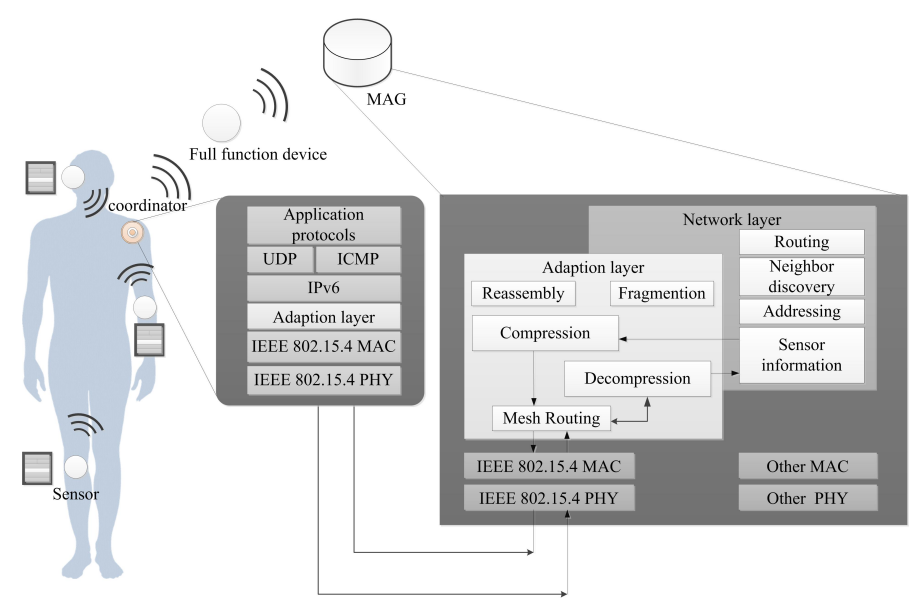

Fig. 1. The protocol stack and system model of Mobile Access Gateway (MAG) and sensors in WBAN

group mobility scheme and a new format of RS and RA message is proposed in this paper to solve those problems.

\section{PRELIMINARIES}

This section introduces the system model first follows by describing the basic idea.

\section{A. System Model}

The proposed protocol is designed for a WBAN consists of 6LoWPAN sensors and a Personal Area Network (PAN) coordinator. Fig 1 illustrates the 6LoWPAN protocol stack of the proposed protocol. 6LoWPAN uses the IEEE 802.15.4 standard as the link-layer protocol. The adaption-layer is used to compress the IPv6 packet header (40 bytes) into 2 bytes for incoming communication (6LoWPAN) and decompress for the outgoing interface (PMIPv6). The 6LoWPAN domain contains full-function devices (FFDs), which support all IEEE 802.15.4 functions and features. The Mobile Body Sensors (MBSs) send the packet through the FFDs to the Mobile Access Gateway (MAG), which has two type interfaces for end-toend communication. The MAG receives the packet, and then decompresses the packet in adaption layer to follow the IPv6 packet format. Thus, the Local Mobility Anchor (LMA) set the binding state for the MBSs after receiving the packet from the MAG. When an MBS attaches to a MAG via wireless link, it sends a Router Solicitation (RS) message which includes the Mobile Node-Identifier (MN-ID) for using an address from its home network prefix (HNP). The MAG emulates the home link of MBS by replying the Router Advertisement (RA) message to the MBS. Thus, the MBS can configure the same address through the HNP in PMIPv6 domain. There is one centralized LMA in the PMIPv6 domain, which acts like a home agent for all mobile nodes. For increasing the coverage of one MAG scope, 6LoWPAN is in beacon-enable mode and equips with the FFDs to support multi-hop communications. Hence, the packet can successfully send to the MAG through multi-hop communications and vice versa. 

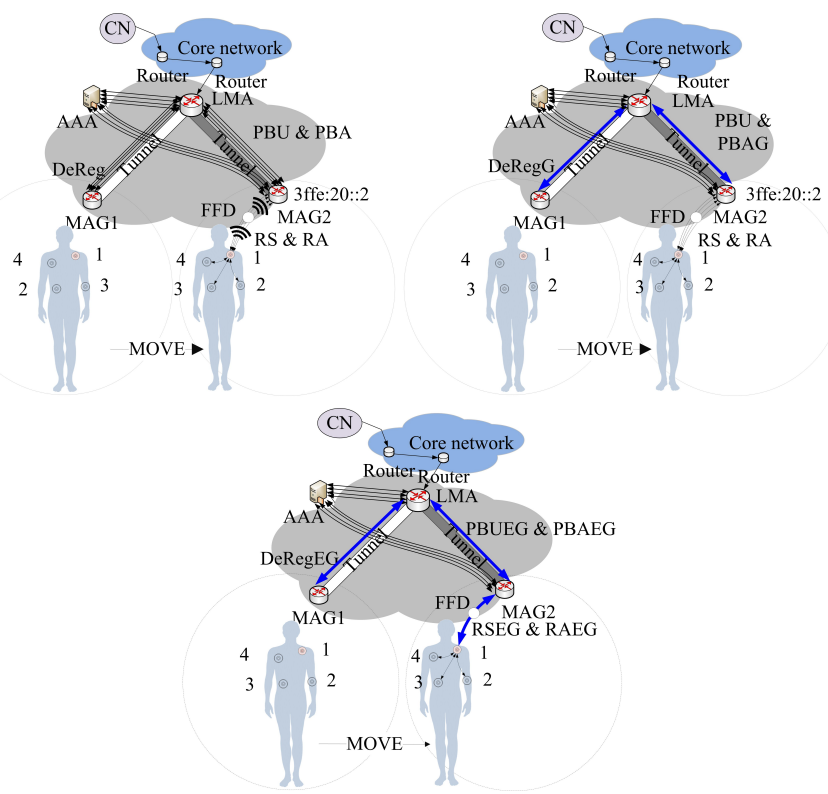

Fig. 2. The comparison among three protocols (a) original protocol (b) group-based protocol(c) proposed protocol.

\section{B. Basic Idea}

The basic idea of this work is to reduce the handoff delay and signaling cost for WBAN roams in PMIPv6. A new format of control message is proposed to combine the necessary information of the sensors into one message and thus the number of control messages can be substantially reduced. Besides, an enhanced group-mobility scheme is proposed to further reduces the handoff delay time between the LMA and the MAG. The signaling cost can also be reduced due to the group management. Fig. 2 illustrates the difference of signaling cost among three protocols. Fig. 2(a) is the original protocol and the protocol showing in Fig. 2(b) is proposed in [9]. The enhanced group protocol is shown in Fig. 2(c). Assuming that there are $n$ sensors attached on a human, who enters or roams in PMIPv6 domain. Fig. 2(a) shows that every kinds of control message have to send $n$ times in the original protocol. Fig. 2(b) illustrates that the group-based protocol can reduce $n-1$ times of sending deregistration of $P B A \mathrm{~s}$ and $P B U \mathrm{~s}$. But it still causes high signaling costs while the first node does not contain the rest nodes' information.

Fig. 3 shows the traffic flow among the original protocol, the group-based protocol, and the proposed protocol. It illustrates the difference on delay time among three protocols. The vertical axis represents the delay time during handoff process. The proposed group-based protocol can reduce the times of sending control messages and thus decreases the delay time.

\section{An Enhanced Group-Based Handoff Scheme}

To achieve the goal of reducing the signaling cost and delay time, three phases are proposed, namely registration, up-link handoff, and down-link handoff phases.

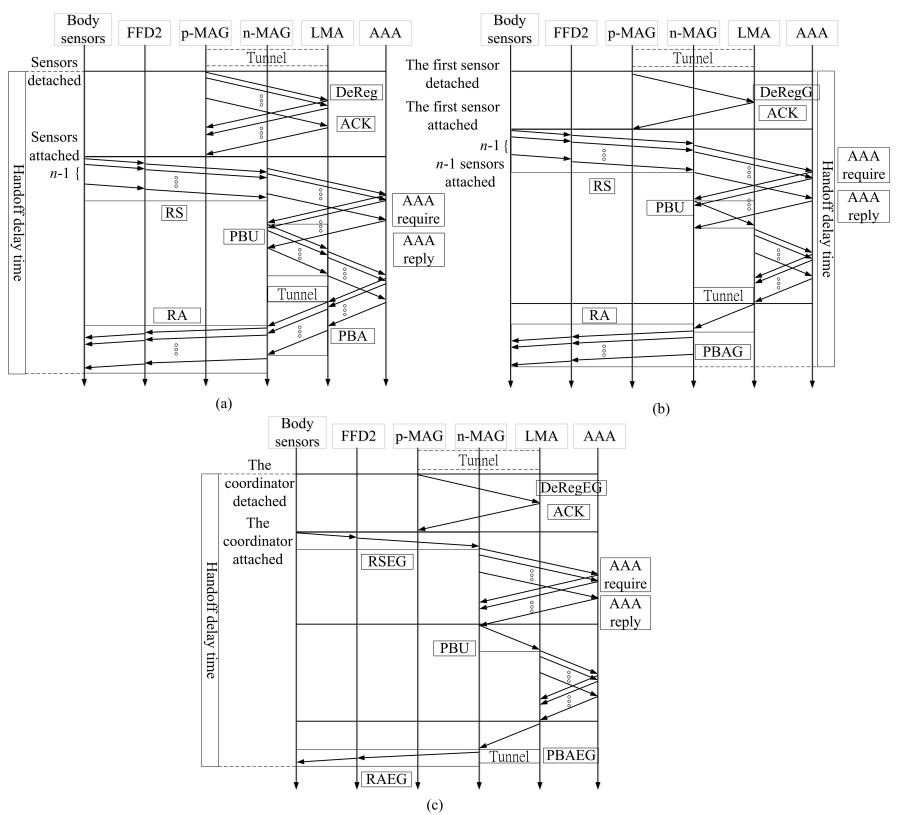

Fig. 3. The comparison of the traffic flow among three protocols (a) original protocol (b) group-based protocol(c) proposed protocol.

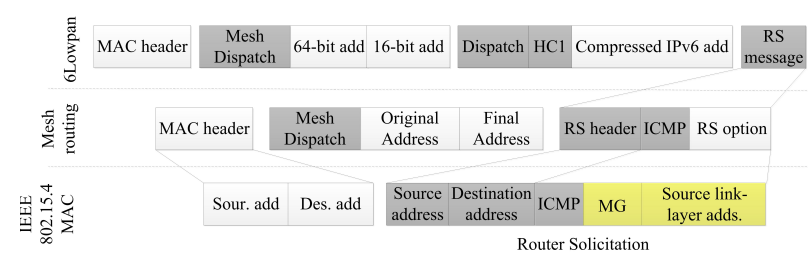

Fig. 4. The packet format of the proposed $R S_{E G}$ message

\section{A. The New Packet Format}

The enhanced mobility protocol mainly simplifies the procedure of home registration and handoff. To achieve this goal, a new format of RS and RA messages is proposed in this paper to reduce the signaling cost and handoff delay. $R S_{E G}$ and $R A_{E G}$ are used to represent the proposed new format of the $R S$ and $R A$ messages, respectively. The $R S_{E G}$ message contains the following components: $<$ Header, ICMP, Body number (BN), $M N_{-} I D_{1}, L L_{-} I D_{1}, M N_{-} I D_{2}, L L_{-} I D_{2}, \ldots$, $M N \_I D_{n}, L L \_I D_{n}>$, where $M N_{-} I D_{i}, L L_{-} I D_{i}$ indicates a set of mobile node-identifiers and link-layer identifiers of $S_{i}$ and $S_{i}$ denotes the $i$-th sensor attached on the human body. The $R S_{E G}$ message contains the following components: $<$ Header, ICMP, $B_{j}, H N P_{1}, H N P_{2}, H N P_{3}, \ldots, H N P_{n}>$, where $B_{j}$ indicates the $j$-th body and $H N P_{i}$ indicates a set of home network's prefixes of sensor $S_{i}$. The packet format of the proposed $R S_{E G}$ message is shown in Fig. 4.

\section{B. The Registration Phase}

The registration phase aims to reduce the amount of control messages. Fig. 5 illustrates the signaling call flow. One of the body sensors needs to act as a coordinator, which can interact with other sensors in 6LoWPAN environment. All the sensors 


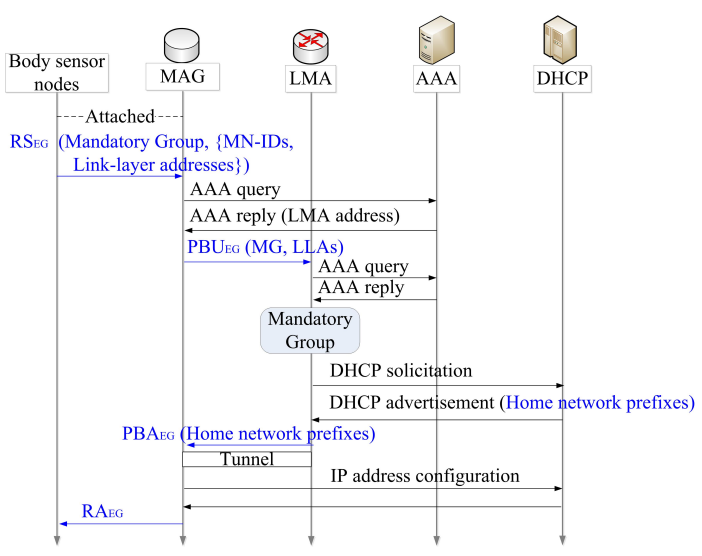

Fig. 5. The signaling call flow for the proposed registration scheme.

use DHCP-based address configuration. The procedure of the group-based registration is given as follows:

S1 When a body sensor enters a PMIPv6 domain and attaches to an access link, the body sensor sends an $R S_{E G}$ message by multi-hop transmissions to the MAG.

S2 Upon the MAG received the $R S_{E G}$ message, the MAG uses all the $L L-I D_{i}$ one by one for authentication by sending the AAA query. After a successful authentication, the AAA server sends a reply which includes the LMA's address and the MN's profile.

S3 After the MAG gets the LMA's address, MAG then sends a $P B U_{E G}$ message, which contains all the identifiers, to the LMA.

S4 Once the LMA receives the $P B U_{E G}$ message, LMA performs access authentication to verify whether $P B U_{E G}$ message is genuine or not.

S5 If the $P B U_{E G}$ message is trustworthy, the LMA initiates the DHCP solicitation procedure to request HNPs for the body sensors one by one. After the delegating router replies the unique HNPs for the body sensors (still one by one), the LMA creates the binding cache entry, stores the prefixes information, and assigns a $\mathrm{BN}$, which is also added in the binding cache for classifying the body sensors into one group. Then, the LMA replies a $P B A_{E G}$ message, which includes all of the body sensors' HNPs and BN, to the MAG. The LMA also sets up its endpoint of the bidirectional tunnel to the MAG.

S6 After the MAG received the $P B A_{E G}$ message, the MAG stores the $\mathrm{BN}$ and requests the addresses from the DHCP server on behalf of the body sensors. The DHCP server then configures the respective Home of address (HoA) from those prefixes and sends it to the MAG.

S7 Upon received those messages, the MAG stores the IP address and sends an $R A_{E G}$ message back.

\section{The Up-Link Handoff Phase}

The body sensor performs an active scan that searches a list of all the available channels by periodically sending a beacon request to all the nearby FFDs. The nearby FFD, that receive the beacon request from the body sensor, advertise a beacon

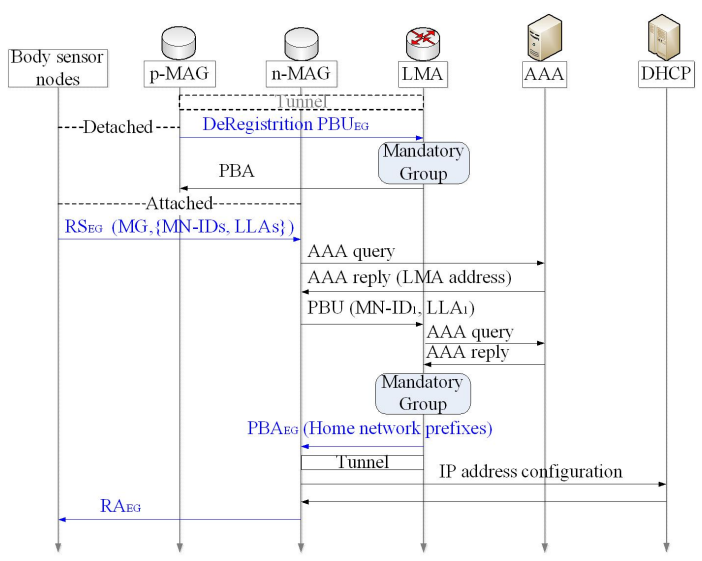

Fig. 6. The signaling call flow for the proposed handoff scheme.

message including their MAG-IDs. Upon receiving the beacon messages, the body sensor decides whether itself is still in the same MAG or has moved to another MAG by comparing the current MAG-ID with the previous MAG-ID contained in the beacon message. If the the comparative results of all the MAG-IDs in the received beacon messages are the same, the movement represents intra-PAN mobility, and the body sensor has moved within the same PAN area. On the other hand, if the body sensor moves from the previous MAG (p-MAG) to the next MAG (n-MAG), the body sensor is able to detect its movement since the current $M A G_{-} I D$ is different from the previous $M A G_{-} I D$, as contained in the received beacon messages. The body sensor can then be associated with the new MAG.

Fig. 6 shows the detail of the signaling call flow for the handoff procedure. The proposed protocol is able to reduce $(i-1)$ RS messages because it replaces per identifier per RS message with a set of all identifiers. Furthermore, by using the assigned $\mathrm{BN}$, it can also save $(i-1)$ handoff messages such as deregistration $P B U, P B U$, and $P B A$. The proposed group-based handoff scheme is described as follows.

S1 When the previous link on MAG (p-MAG) detects the detachment event from the body sensor, the MAG will signal the LMA by sending the deregistration $P B U$. Instead of all nodes sending the deregistration $P B U$ respectively (as the original protocol did), here the MAG sends only one $L L_{-} I D$ from the body sensor. Upon the LMA receives this message, LMA can obtain the rest sensors' information due to previously assigned BN, which is stored in the binding cache. After the LMA has confirmed all the information of sensors, the LMA will remove the binding and routing states if the LMA does not receive any $P B U$ message within the given amount time. Then, the LMA replies a $P B A$ message and informs the MAG not to send other unnecessary deregistration $P B U$ messages. This way, the proposed scheme can reduce $(n-1)$ times of sending deregistration $P B U$ to advertise the LMA.

S2 In order to let the n-MAG obtains the assigned group 
value of the body sensor, once the body sensor attaches to the new MAG (n-MAG), the coordinator of the body sensor sends the $R S_{E G}$ message on behalf of all sensors to the n-MAG by unicast.

S3 Upon the n-MAG received the $R S_{E G}$ message, MAG stores the $\mathrm{BN}$, all the $M N-I D_{i}$ and $L L_{-} I D_{i}$ in the binding cache. Then the n-MAG uses all the $L L_{-} I D_{i}$ one by one for authentication by sending the AAA query. After a successful authentication, the AAA server sends a reply which includes the LMA's address and the MN's profiles.

S4 This step aims to enhance the handoff performance by using the assigned group value in the LMA. Therefore, After the n-MAG obtaining the LMA's address, nMAG sends a $P B U_{E G}$ message Compare to the original PMIPv6 protocol, the $P B U_{E G}$ message can reduce $(n-1)$ times of binding message. The reason is that the LMA can obtain the other sensors' binding information by using the previously assigned $\mathrm{BN}$.

\section{Down-Link Handoff Phase}

This phase aims to reduce the handoff delay and signaling cost during the handoff period. The details of this phase are described as follows:

S1 Once the LMA received the $P B U_{E G}$ message, the LMA still needs to perform authentication to verify whether the $P B U_{E G}$ message is genuine or not.

S2 For obtaining the whole assigned prefixes information of the body sensor, the LMA uses the classified group ID to search for the necessary data from the binding cache. Hence, if the $P B U_{E G}$ is trustworthy, within the given amount of time, the LMA replies a $P B A_{E G}$ message, which includes all the body sensor's unique $H N P_{i}$. The LMA also changes the bidirectional tunnel from the pMAG to the n-MAG and stored in the binding cache entry.

S3 After the n-MAG received the $P B A_{E G}$ message, the $\mathrm{n}$ MAG stores the information of HNPs and then sends the DHCP requests. Thus, the DHCP server identifies the client from the client-DUID and will identify that link from the link-address. After that, the DHCP server will allocate the same addresses of all the body sensors' prefixes respectively to the n-MAG one by one.

S4 Upon received IP configuration messages, the n-MAG stores the IP address and sends an $R A_{E G}$ message to the coordinator of the body sensor. After the coordinator receiving the $R A_{E G}$ message, the coordinator will broadcast the ACK to the rest of the body sensors.

\section{Simulation Results}

To evaluate the proposed enhanced group mobility protocol and the group-based handoff scheme [11], the Network Simulator-2 (NS2) with 6LoWPAN and PMIPv6 modules is used to simulate these protocols. The networks size is $150 \times 150 \mathrm{~m}^{2}$, the packet size is 1000 bytes and the data rate is $250 \mathrm{kbps}$. The initial delay value of the wireless link delay is $10 \mathrm{~ms}$ and the delay link between the corresponding
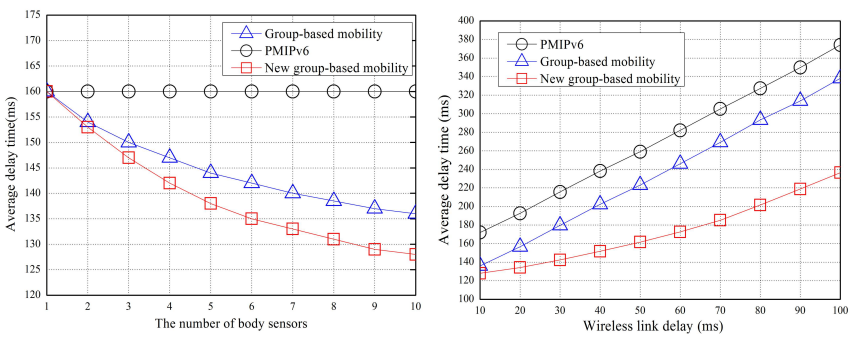

Fig. 7. Performance of average delay time vs. (a) The number of sensors. (b) Wireless link delay.

node and LMA is set as $10 \mathrm{~ms}$. The simulation scenario is that a human, who has attached several sensor nodes (from 1 to 10), moves from left to right. In this situation, the sensors implement the handoff process at the same time. The packet is forwarded from p-MAG to n-MAG. The performance metrics to be observed are shown as follows:

- The average delay time (ADT) is defined as the total delay time divided by the total number of sensor nodes.

- The packet loss ratio (PLR) is defined as the total number of lost data packets divided by the total number of transmitted data packets.

- The average signaling cost (ASC) is defined as the total number of control messages divided by the total number of sensor nodes for performing the handoff procedure.

- The packet overhead (PO) is defined as the total number of control packets and all data packets which includes retransmitted packets.

The simulation results for average delay time (ADT), packet loss ratio (PLR), average signaling cost (ASC), and packet overhead (PO) are discussed from several aspects in following subsections.

\section{A. Average Delay Time (ADT)}

The simulation results of Average Delay Time (ADT) under the number of body sensors and wireless link delay are illustrated in Fig 7. Fig. 7(a) shows the number of body sensors (from 1 to 10 sensors) vs. the wireless link delay (ranging from 10ms to $100 \mathrm{~ms}$ ). In general, the ADT drops as the data size increases. This is because that less control messages are used. Fig. 7(b) provides the simulation result of the average handoff latency (ADT) vs. the wireless link delay (ranging from $10 \mathrm{~ms}$ to $100 \mathrm{~ms}$ ). The ADT increases as the velocity increases because higher moving speed incurs higher frequency of handoff.

\section{B. Packet Loss Ratio (PLR)}

The simulation results of the packet loss ratio (PLR) under the number of sensors and wireless delay time are illustrated in Fig 8. Fig. 8(a) shows the simulation result of PLR vs. the number of body sensors (ranging from 1 to 10 sensors). The packet loss occurs when the handover is executed. In general, the PLR increases as the number of sensors increases. 

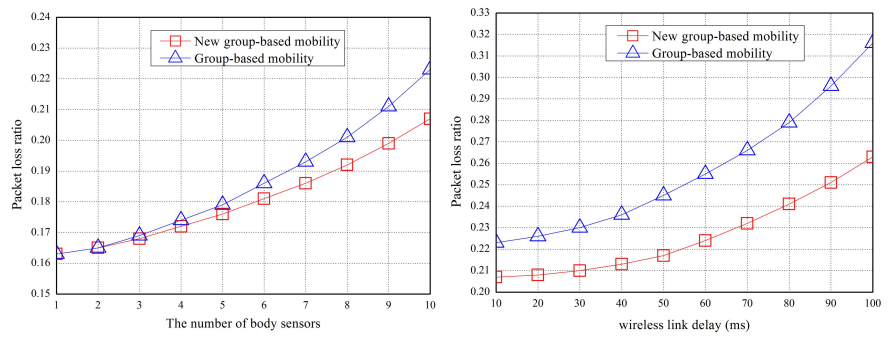

Fig. 8. Performance of packet loss ratio vs. (a) The number of sensors. (b) Wireless link delay.
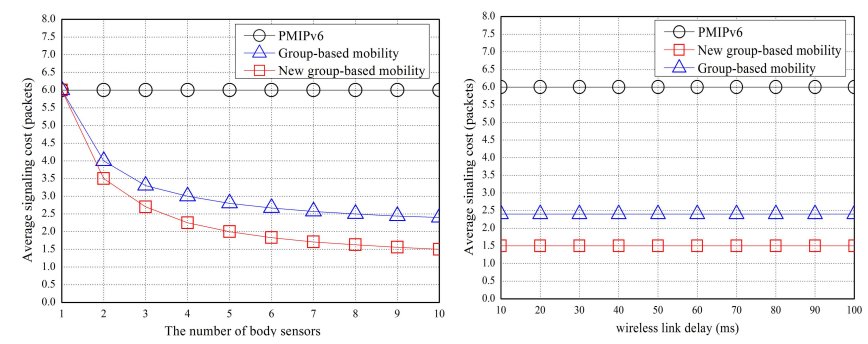

Fig. 9. Performance of average signaling cost vs. (a) The number of sensors. (b) Wireless link delay.

The PLR of our protocol is smaller than that of the groupbased protocol with the increasing number of body sensors. Fig. 8(b) shows the simulation result of the PLR vs. the wireless link delay (ranging from $10 \mathrm{~ms}$ to $100 \mathrm{~ms}$ ). In general, the PLR increases as the wireless link delay increases. The PLR of our protocol is smaller than group-based protocol with wireless link delay increasing. The reason is that the number of exchanged RS and RA messages in our enhance group mobility protocol is smaller.

\section{Average Signaling Cost (ASC)}

Fig. 9 shows the simulation results of the average signaling cost of handooff (ASC). Fig. 9(a) shows the simulation result of the ASC vs. the number of body sensors (ranging from 1 to 10 sensors). The ASC indicates the number of message exchanging between sensor nodes and MAG. The ASC of the proposed scheme is smaller than group-based scheme because the proposed protocol can reduce the control message by using one message to carry other sensors nodes' information. In general, the ASC decreases as the number of body sensor increases. Fig. 9(b) offers the simulation result of the ASC vs. the wireless link delay (ranging from $10 \mathrm{~ms}$ to $80 \mathrm{~ms}$ ). In general, the ASC increases but does not have the direct relationship with the wireless link delay.

\section{Packet Overhead (PO)}

The simulation results of Packet Overhead (PO) under the number of sensors and wireless delay time are illustrated in Fig. 10. Fig.10(a) shows the PO vs. the number of body sensors (ranging from 1 to 10 sensors). In general, the PO increases as the number of body sensors increases. This is
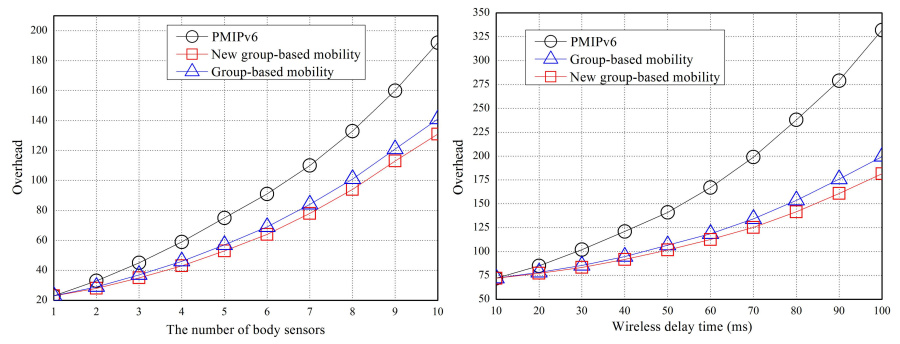

Fig. 10. Performance of overhead vs. (a) The number of sensors. (b) Wireless link delay.

because there is less control messages during the handoff procedure. Fig.10(b) provides the simulation results of the packet overhead (PO) vs. the wireless link delay (ranging from 1 to 10 sensors). The PO increases as the wireless link delay increases.

\section{Conclusions}

In this paper, an enhanced group mobility protocol is proposed. A new format of control message is proposed to combine the necessary information of the sensors into one message and thus reduces the number of control messages. Besides, an enhanced group-mobility scheme is proposed to further reduces the handoff delay time between the LMA and the MAG. The signaling cost can also be reduced due to the group management. Simulation results have shown that the handoff delay and signaling cost can be reduced by using the proposed enhanced group handoff scheme.

\section{ACKNOWLEDGEMENT}

This research was supported by the National Science Council of the R.O.C. under grant NSC-101-2219-E-305-001 and NSC-100-2221-E-305-001-MY3.

\section{REFERENCES}

[1] J. Xing, "A survey on body area network, networking and mobile computing," in Proceedings of International Conference on Wireless Communications, September 2009, pp. 1-4.

[2] "Ipv6 over low power wpan (active wg)," http://tools.ietf.org/wg/6lowpan.

[3] D. Saha, A. Mukherjee, I.S.Misra, and M. Chakraborty, "Mobility support in ip: A survey of related protocols," IEEE Network, pp. 34-40, 2004.

[4] "Hierarchical mipv6 mobility management," RFC 4140, August 2005.

[5] "Proxy mobile ipv6," https://merlot.tools.ietf.org/html/rfc5213, August 2008.

[6] R.Istepanian, E. Jovanov, and Y. Zhang, "Guest editorial introduction to the special section on mhealth: Beyond seamless mobility and global wireless health-care connectivity," IEEE Transactions on Information Technology in Biomedicine, pp. 405-414, 2004.

[7] G. Kushalnagar, G. Montenegro, J. Hui, and D. Culer, "Transmission of ipv6 packets over ieee 802.15.4 networks," IETF RFC 4944, 2007.

[8] J. H. Kim, C. S. Hong, and T. Shon, "A lightweight nemo protocol to support 6lowpan," ETRI Journal, pp. 685-695, 2008.

[9] V. Devarapalli, R. Wakikawa, A. Petrescu, and P. Thubert, "Network mobility (nemo) basic support protocol," IETF RFC 3963, 2005.

[10] Y. Li, Y. Jiang, H. Su, D. Jin, L. Su, and L. ZengA, "A group-based handoff scheme for correlated mobile nodes in proxy mobile ipv6," in Proceedings of IEEE Global Telecommunications Conference, December 2009, pp. 1-6.

[11] M. M. Islam and E. N. Huh, "A novel addressing scheme for pmipv6 based global ip-wsns," Sensors, vol. 11, pp. 5844-5877, August 2011. 